\title{
UCHL1 acts as a colorectal cancer oncogene via activation of the $\beta$-catenin/TCF pathway through its deubiquitinating activity
}

\author{
JIALING ZHONG ${ }^{1}$, MEI ZHAO ${ }^{1}$, YIMING MA ${ }^{1}$, QING LUO ${ }^{1}$, JIAN LIU $^{1}$, JIA WANG $^{1}$, \\ XINGHUA YUAN ${ }^{2}$, JIANLI SANG ${ }^{3}$ and CHANGZHI HUANG ${ }^{1}$
}

\begin{abstract}
${ }^{1}$ Department of Etiology and Carcinogenesis and State Key Laboratory of Molecular Oncology, and ${ }^{2}$ Department of Abdomen Surgery, Cancer Institute and Hospital, Chinese Academy of Medical Sciences and Peking Union Medical College, Beijing 100021; ${ }^{3}$ Institute of Cell Biology, College of Life Sciences, Beijing Normal University, Beijing 100875, P.R. China
\end{abstract}

Received March 6, 2012; Accepted May 3, 2012

DOI: $10.3892 /$ ijmm.2012.1012

\begin{abstract}
Ubiquitin C-terminal hydrolase-L1 (UCHL1) belongs to the family of deubiquitinating enzymes (DUBs), which is involved in the ubiquitin-dependent proteolytic system. Previously, we have reported that the upregulation of UCHL1 is related to lymph node metastasis in colorectal cancer (CRC). However, its molecular mechanisms remain elusive. In this study, we transfected pcDNA3.1/UCHL1 and the pcDNA3.1/UCHL1-C90S mutant into HCT8 cells. The changes in biological features in these stable transfectants were examined both in vitro and in vivo. Western blot analysis was used to analyze the changes in the $\beta$-catenin/T cell factor (TCF) pathway. We demonstrated that UCHL1 re-expression promoted the proliferation, migration and metastasis potential of HCT8 cells both in vitro and in vivo. We also found that UCHL1 could decelerate $\beta$-catenin degradation depending on its deubiquitinating activity. The accumulated $\beta$-catenin consequently activated the $\beta$-catenin/TCF pathway and induced the expression of cyclin D1 and uPA. These observations imply that UCHL1 may contribute to CRC progression by activating the $\beta$-catenin/TCF pathway through its deubiquitinating activity.
\end{abstract}

\section{Introduction}

Colorectal cancer (CRC) is the third most common cancer, and the fourth leading cause of cancer death worldwide (1). In recent years, improved treatment has resulted in a 5-year survival rate of about $50 \%$ (2). However, the survival rate of

Correspondence to: Dr Changzhi Huang, Department of Etiology and Carcinogenesis and State Key Laboratory of Molecular Oncology, Cancer Institute and Hospital, Chinese Academy of Medical Sciences and Peking Union Medical College, 17 Panjiayuan Nanli, Chaoyang, Beijing 100021, P.R. China

E-mail: huangpumc@163.com

Key words: ubiquitin C-terminal hydrolase-L1, colorectal cancer, $\beta$-catenin/T cell factor pathway advanced stage patients, especially of those with lymph node metastasis, is still low. A series of genes are involved in the pathogenesis of CRC. Investigation of these genes is important to clarify the tumorigenic pathway and is helpful for developing new biomarkers for early detection and accurate diagnosis.

Ubiquitin C-terminal hydrolase-L1 (UCHL1) is a member of the deubiquitinating enzymes (DUBs), widely expressed in neuronal tissues, and functions to maintain ubiquitin balance by associating and releasing ubiquitin (3). Previous studies showed that UCHL1 played an important role in neurodegenerative diseases including Parkinson's disease and Alzheimer's disease (4). Recently, growing evidence indicates that UCHL1 also plays a role in tumorigenesis and tumor progression. Interestingly, the roles of UCHL1 are conflicting in different cancer types. UCHL1 acts as a tumor promoter in pancreatic cancer, prostate cancer, and lung cancer (5-7). However, it acts as a tumor suppressor in ovarian cancer, hepatocellular cancer, and nasopharyngeal carcinoma (8-10). In esophageal cancer, breast cancer and CRC, its function is ambiguous (10,11-15). The role of UCHL1 in cancer cells is still largely unexplored.

In our previous study, we found that the expression of UCHL1 was increased in the lymph node metastasis (LNM) CRC group compared with the non-LNM CRC group. The re-expression of UCHL1 through gene transfection increased the invasive ability of HCT8 cells (16). In this study, we further demonstrated that UCHL1 re-expression promoted the proliferation, migration, and metastatic capabilities of HCT8 cells both in vitro and in vivo. We also found that UCHL1 could decelerate $\beta$-catenin degradation depending on its deubiquitinating activity. Accumulated $\beta$-catenin consequently activated the $\beta$-catenin/T cell factor (TCF) pathway and induced the expression of cyclin D1 and uPA.

\section{Materials and methods}

Cell culture and transfection. HCT8 cells were maintained in Roswell Park Memorial Institute (RPMI)-1640 (Invitrogen, USA) supplemented with $10 \%$ heat-inactivated fetal bovine serum (FBS) at $37^{\circ} \mathrm{C}$ in a humidified $5 \% \mathrm{CO}_{2}$ atmosphere. DNA transfection was performed using Lipofectamine (Invitrogen) according to the manufacturer's protocol. For stable transfection, cells were selected using full growth medium containing 
G418 $(600 \mu \mathrm{g} / \mathrm{ml})$ for 14 days, and the positive cells were detected by western blot analysis.

Expression plasmids. Wild-type pcDNA3.1/UCHL1 expression plasmid against UCHL1 was a gift from Dr Julia Shackelford. The pcDNA3.1/UCHL1-C90S mutant was generated by inserting a specific mutation at the Cys 90 site in wild-type pcDNA3.1/UCHL1 plasmid by overlap PCR.

Cell growth assay by MTT. Cells were seeded in triplicate into 96-well plates with $3 \times 10^{3}$ cells/well. Cells were maintained for 5 days, and then $20 \mu \mathrm{l}$ of MTT (Sigma, USA) solution $(5 \mathrm{mg} / \mathrm{ml})$ was added and cells were further incubated at $37^{\circ} \mathrm{C}$ for $4 \mathrm{~h}$. Absorbance at $492 \mathrm{~nm}$ was determined with a microplate reader. All MTT assays were repeated three times.

In vitro cell invasion and migration assays. Cell invasion and migration assays were carried out in a chemotaxis chamber (Millipore). Cells $\left(1 \times 10^{5}\right)$ suspended in $200 \mu \mathrm{l}$ of serum-free medium were loaded into the upper chamber and allowed to pass through an $8-\mu \mathrm{m}$-pore polyethylene terephthalate filter. The lower chamber was filled with complete medium. The chamber was either pre-coated with Matrigel (1:8; BD Biosciences, Bedford, MA, USA) for invasion assays or left uncoated for migration assays. Cells that failed to pass through the filters were removed by scrubbing with cotton swabs after $20 \mathrm{~h}$ (migration assay) or $24 \mathrm{~h}$ (invasion assay). Cells on the undersurface were fixed in methanol and stained with $0.5 \%$ crystal violet, then photographed and quantified in 5 random fields per membrane. Each sample was assayed in triplicate.

Tumor growth and metastasis in nude mice. For the tumor growth assay, cells ( $40 \mu 1,4 \times 10^{6}$ cells) were collected and inoculated subcutaneously into the nail pad of 4 -week-old male nude mice. Experimental and control groups had 5 mice each. Mice were sacrificed 38 days later. Tumor growth curves were calculated. After tumor excision, the tissues were fixed in $10 \%$ buffered formalin. All formalin-fixed and paraffinembedded samples were carefully examined after staining with hematoxylin and eosin (H\&E) and UCHL1 antibody.

For the tumor metastasis assay, cells (50 $\mu 12.5 \times 10^{6}$ cells) were collected and injected into the tail vein of 4-week-old male nude mice. Each group included 6 mice. Mice were sacrificed 4 weeks later and examined for metastasis development. Lungs and visibly observable lymph nodes were excised. All lungs were fixed with Bouin's fluid. All lymph nodes were formalin-fixed and paraffin-embedded, then carefully examined after staining with H\&E and UCHL1 antibody.

All animal experiments were conducted according to the institutional and national guidelines for animal experiments.

Immunohistochemical staining. Immunohistochemical (IHC) staining was performed as previously described (16). Specimens were incubated with anti-UCHL1 (1:500, Abcam) overnight at $4^{\circ} \mathrm{C}$, and then incubated with a biotinylated second antibody (goat anti-rabbit/IgG 1:100 dilution) for $30 \mathrm{~min}$ at $37^{\circ} \mathrm{C}$.

Half-life analysis. Cells were treated with cyclohexamide (CHX, $100 \mu \mathrm{g} / \mathrm{ml}$; Sigma) for 0,3 or $6 \mathrm{~h}$ to inhibit further protein synthesis. Then cells were harvested and analyzed by western blot analysis.

Protein preparation and western blot analysis. For wholecell protein extraction, cells were lysed with lysis buffer [50 mmol/1 Tris-HCl, pH 7.4; 150 mmol/1 NaCl; 1.5\% NP-40; $0.1 \% \mathrm{SDS} ; 50 \mu \mathrm{g} / \mathrm{ml}$ phenylmethylsulfony fluoride (PMSF); with freshly added proteinase inhibitor cocktail] for $40 \mathrm{~min}$ on ice, followed by centrifugation at $14,000 \mathrm{x} \mathrm{g}$ for $15 \mathrm{~min}$ at $4^{\circ} \mathrm{C}$. The supernatants were collected as total protein.

For preparation of cytoplasmic and nuclear protein, cells were collected and rinsed with cold phosphate-buffered saline (PBS) twice, then centrifuged at $3000 \mathrm{xg}$ for $5 \mathrm{~min}$ at $4^{\circ} \mathrm{C}$. The pellets were suspended in buffer A (10 mM HEPES, $\mathrm{pH} 7.9$; $1.5 \mathrm{mM} \mathrm{MgCl}$; $10 \mathrm{mM} \mathrm{KCl} ; 0.5 \mathrm{mM}$ DTT; 0.2\% NP-40; $50 \mu \mathrm{g} / \mathrm{ml}$ PMSF; with freshly added proteinase inhibitor cocktail) and kept for $15 \mathrm{~min}$ at $4^{\circ} \mathrm{C}$, then centrifuged at $5,000 \mathrm{x} \mathrm{g}$ for $5 \mathrm{~min}$. The supernatants (cytoplasmic protein) were harvested. The insoluble pellets were then centrifuged at $14,000 \mathrm{x}$ g for $1 \mathrm{~min}$, again after being washed with cold buffer $\mathrm{A}$. The pellets were lysed in lysis buffer and kept for $40 \mathrm{~min}$ at $4^{\circ} \mathrm{C}$, then centrifuged at $14,000 \mathrm{x} \mathrm{g}$ for $10 \mathrm{~min}$. The supernatants were harvested as nuclear protein. Western blot analysis was performed using conventional protocols as described previously (16).

Statistical analysis. All statistical analyses were performed using SPSS 13.0 software (SPSS Inc., Chicago, IL, USA). Data were expressed as means \pm SD. The Student's t-test was used for statistical comparison. P-values $<0.05$ indicated statistical significance.

\section{Results}

UCHL1 promotes proliferation, migration and invasion capabilities of HCT8 cells in vitro. As we previously reported (16), HCT8 did not express endogenous UCHL1. Thus, we used HCT8 to generate the UCHL1 stable cell line. The HCT8/ UCHL1 stable cells expressed high exogenous UCHL1, and the HCT8/pcDNA3.1 cells were used as empty vector control (Fig. 1A). HCT8/UCHL1 cells showed increased cell proliferation, migration, and invasion capabilities in vitro compared with control cells $(\mathrm{P}<0.05)$ (Fig. 1B-D). These results implied that UCHL1 promoted the proliferation, migration, and invasion capabilities of HCT8 cells in vitro.

UCHL1 promotes tumorigenesis and metastasis of HCT8 cells in vivo. To investigate UCHL1 functions in vivo, we injected HCT8/pcDNA3.1 and HCT8/UCHL1 cells into nude mice through the nail pad. Mice were humanely sacrificed and dissected for tumors 38 days later. Tumor nodules resected from the HCT8/UCHL1 group showed positive UCHL1 staining (Fig. 2D). In addition, the nodules from this group were larger and heavier than those from the control group $(\mathrm{P}<0.05)$ (Fig. 2A-C). However, we did not find lymph node metastasis or lung metastasis in either group in this model.

To investigate whether UCHL1 promotes tumor metastasis in vivo, we injected HCT8/pcDNA3.1 and HCT8/UCHL1 cells into nude mice via tail veins. Mice were sacrificed 4 weeks later. In the HCT8/UCHL1 group, 2 mice died before the 
A

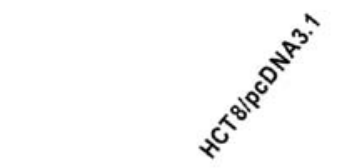

UCHL1-

$\beta$-actin .

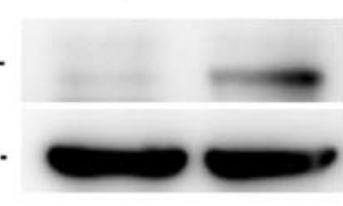

C

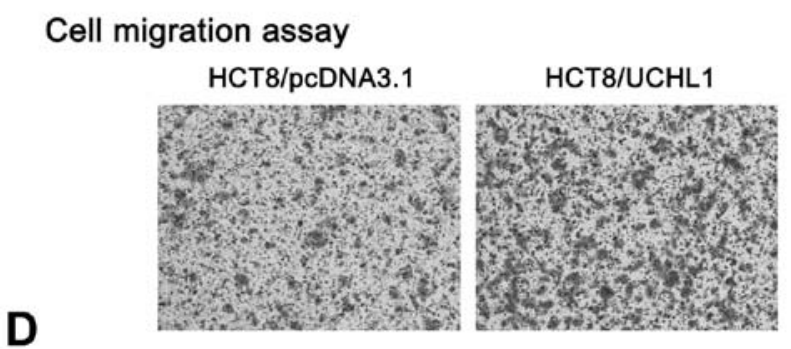

D

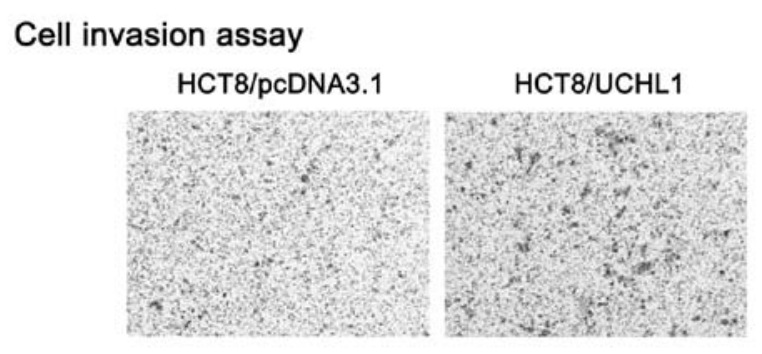

B
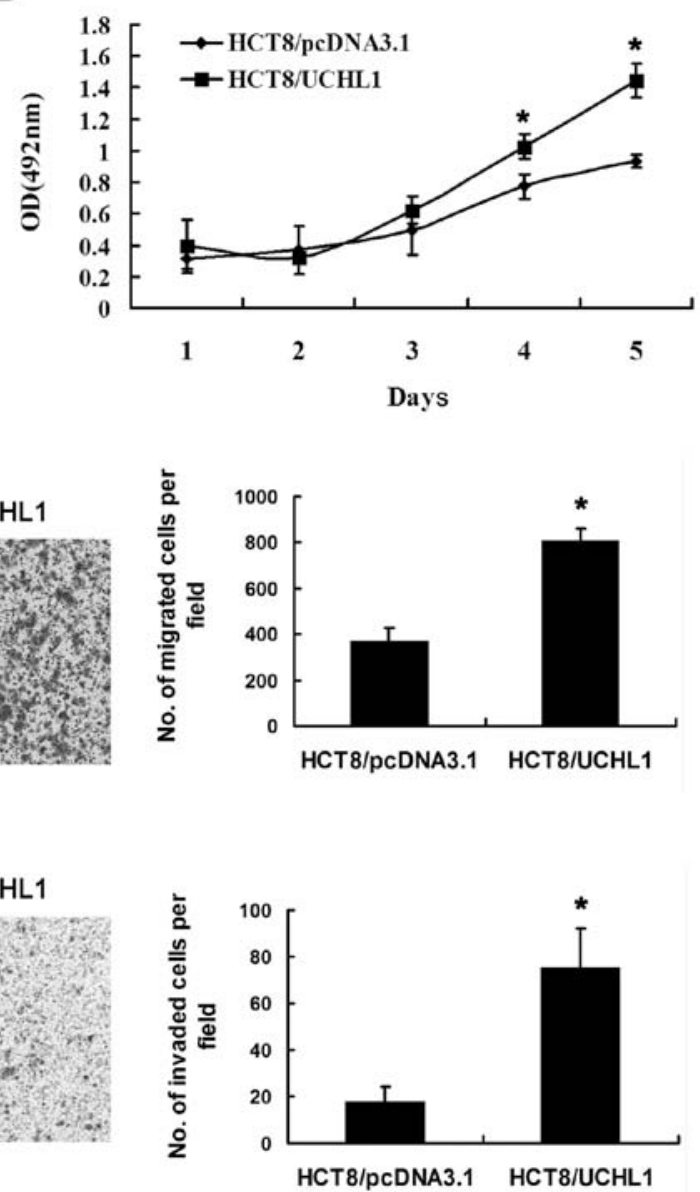

Figure 1. UCHL1 promotes proliferation, migration and invasion capabilities of HCT8 cells in vitro. (A) HCT8 cells were transfected with UCHL1 expression vector or empty vector and maintained in the presence of G418 for 14 days. UCHL1 expression was identified by western blot analysis; $\beta$-actin served as control for sample loading. (B) Cell growth curve measured by the MTT assay. Each point represents optical density \pm SD of three independent experiments. (C) Cell migration and (D) invasion assays were performed by using chemotaxis chambers. Cell number quantified data were shown as means \pm SD $(n=3)$ (magnification, x100). ${ }^{*} \mathrm{P}<0.05$, Student's t-test.

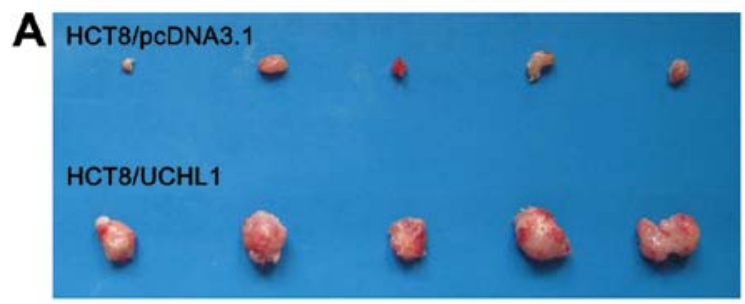

C

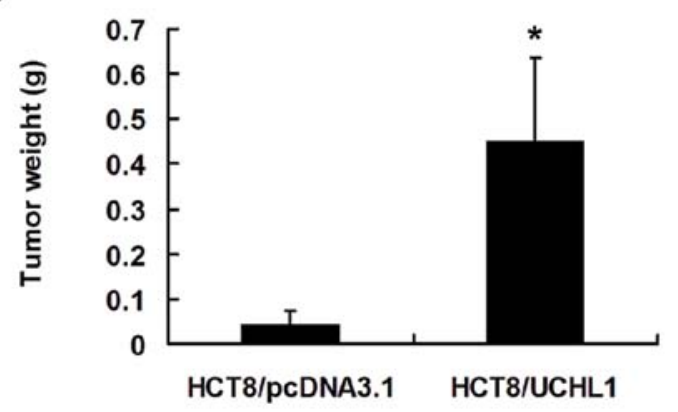

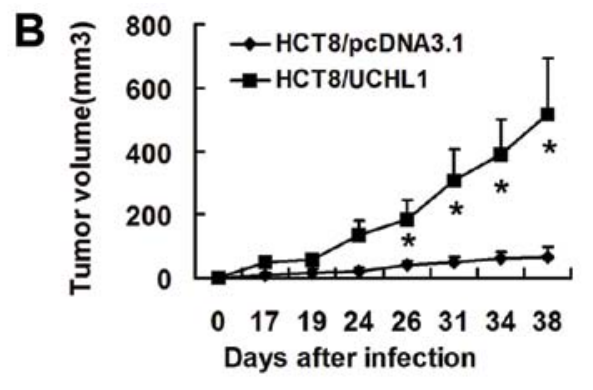

D

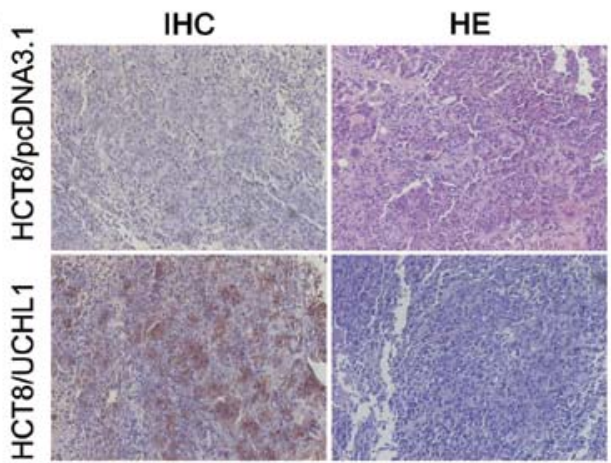

Figure 2. UCHL1 promotes tumor xenografts of HCT8 cells in nude mice. (A) Gross observation of tumor xenografts in different groups. (B) Tumor volume after injection in nude mice. Each point represents tumor volume \pm SD. (C) Tumor weight in different groups at the end of the study. Data are expressed as means \pm SD. (D) IHC and H\&E staining of tumor xenografts in different groups (magnification, $x 100$ ). ${ }^{*} \mathrm{P}<0.05$, Student's t-test. 
A

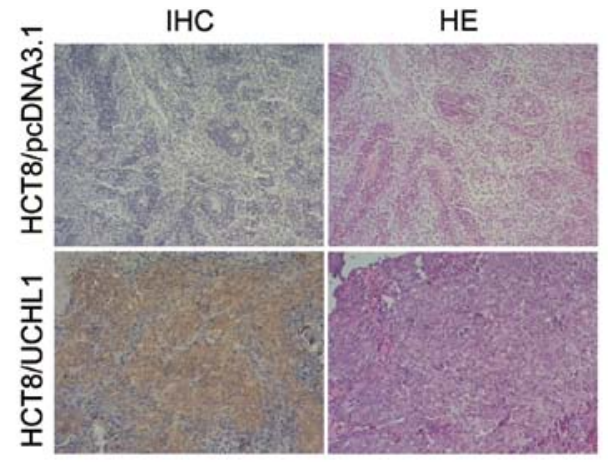

C

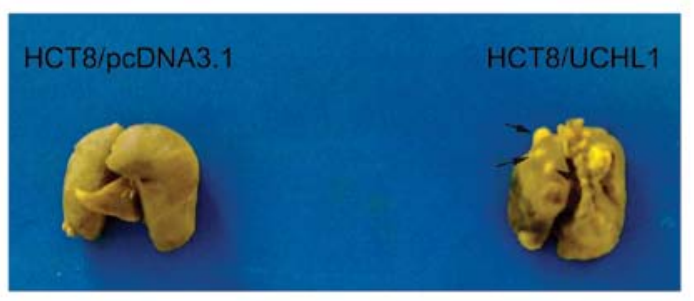

B

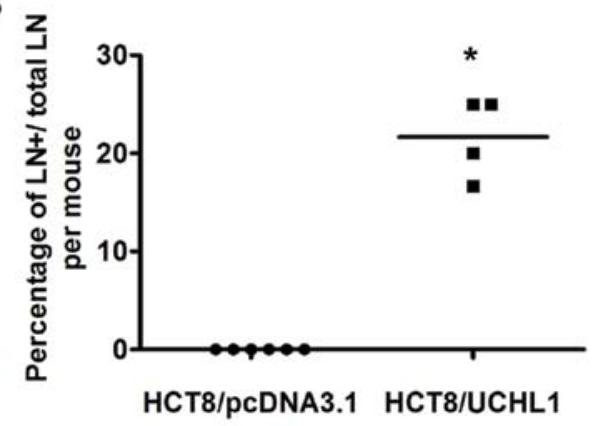

D

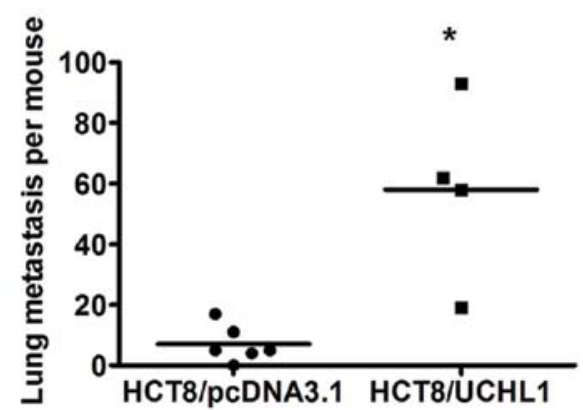

Figure 3. UCHL1 promotes lymph node and lung metastases in nude mice. (A) UCHL1 expression was assessed by immunohistochemistry; H\&E was used for histological examination to confirm metastasis. Top row, normal lymph node resected from the HCT8/pcDNA3.1 group showing negative UCHL1 staining. H\&E staining depicting normal histological structure. Bottom row, metastatic lymph node resected from the HCT8/UCHL1 group showing strong UCHL1 staining. H\&E staining depicting destroyed lymph node histologic structure (magnification, x100). (B) Percentage of metastatic lymph nodes $\left(\mathrm{LN}^{+}\right) / \mathrm{total}_{\mathrm{LN}}$ per mouse. (C) Lung metastases of each group at 4 weeks after injection. (D) Statistical plot showing the metastatic loci counted on the whole lung surface per mouse. ${ }^{*} \mathrm{P}<0.05$, Student's t-test.

A

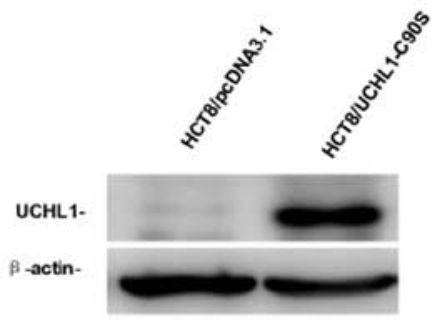

C

Cell migration assay

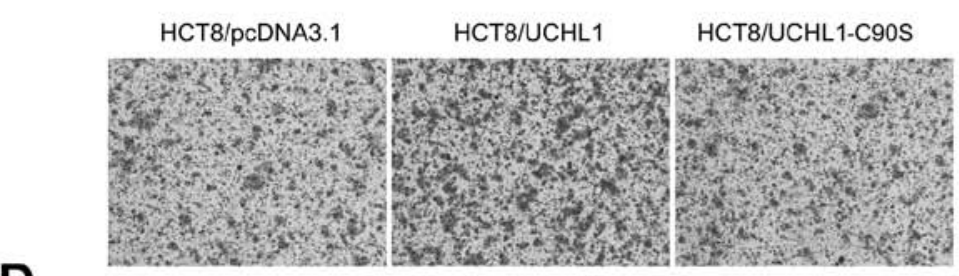

Cell invasion assay

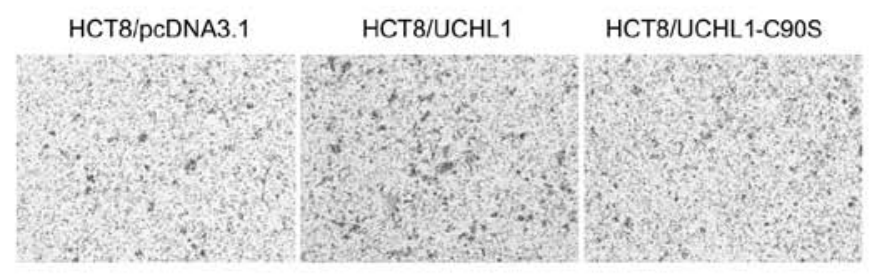

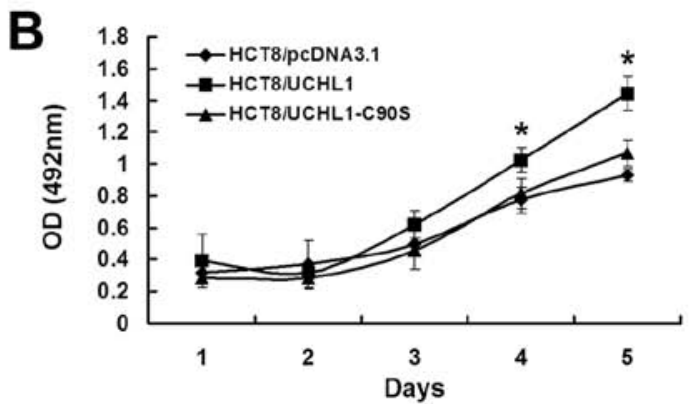
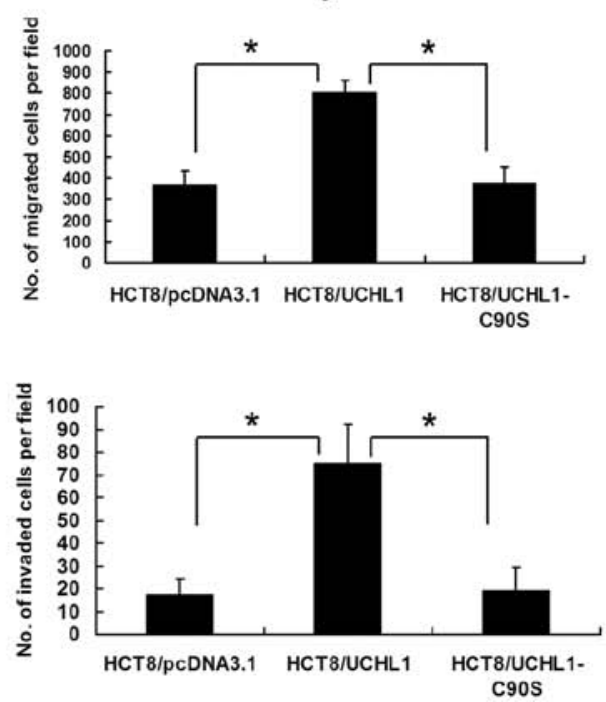

Figure 4. Deubiquitinase activity is crucial for UCHL1 to promote the malignant transformation of HCT8 cells. (A) HCT8 cells were transfected with UCHL1C90S expression vector or empty vector and maintained in presence of G418 for 14 days. UCHL1 expression was identified by western blot analysis; $\beta$-actin served as a control for sample loading. UCHL1-C90S mutant could not promote (B) proliferation, (C) migration and (D) invasion capabilities of HCT8 cells in vitro. ${ }^{*} \mathrm{P}<0.05$, Student's t-test. 
A

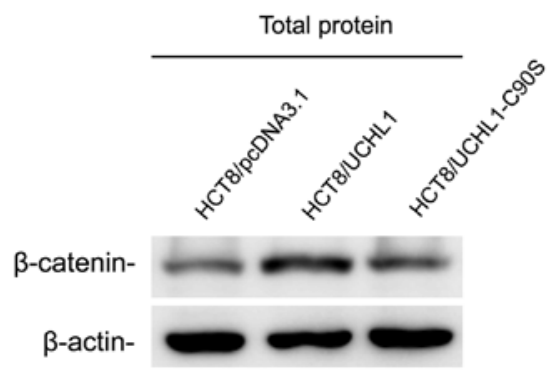

B
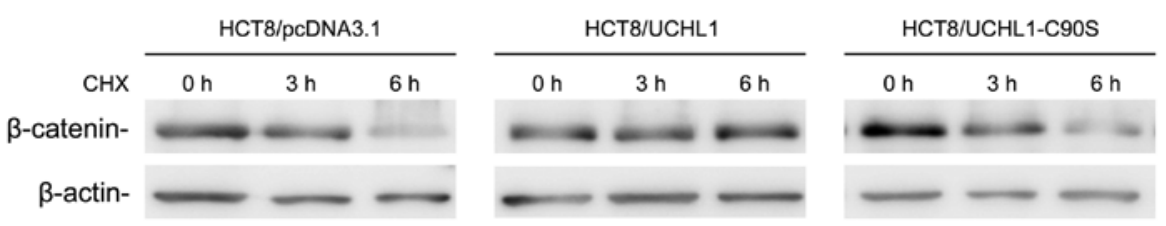

Figure 5. UCHL1 upregulates $\beta$-catenin by enhancing its stability. (A) $\beta$-catenin increased in HCT8/UCHL1 cells compared with HCT8/pcDNA3.1 and HCT8/ UCHL1-C90S cells. (B) Cells were exposed to CHX for different durations. $\beta$-catenin protein levels were evaluated by western bolt analysis. $\beta$-actin served as a loading control.

A

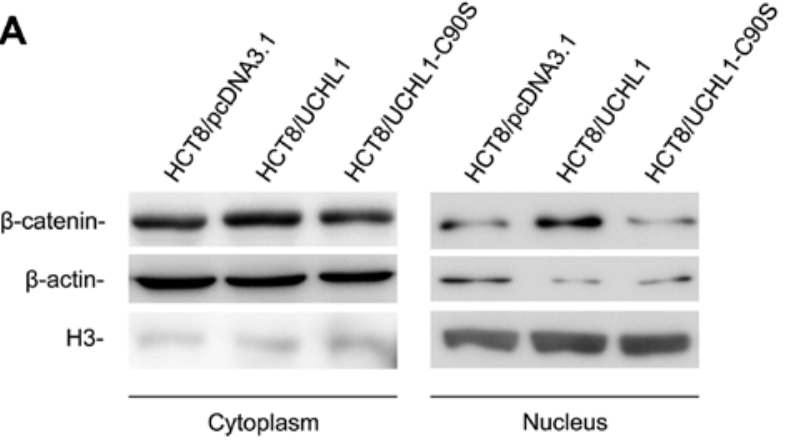

B

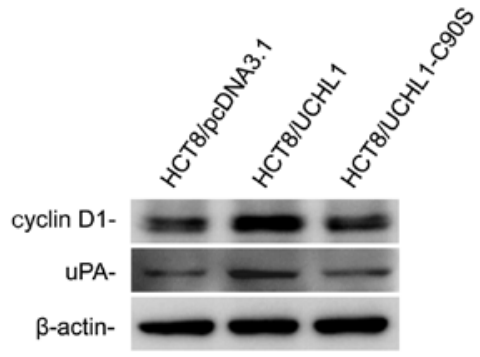

Figure 6. UCHL1 induces the accumulation of $\beta$-catenin in nucleus, and induces the expression of cyclin D1 and uPA. (A) The cytoplasmic and nuclear proteins were isolated from cells and subjected to western blot analysis. $\beta$-catenin accumulated in the nucleus in HCT8/UCHL1 cells compared with HCT8/pcDNA3.1 and HCT8/UCHL1-C90S cells. $\beta$-actin and H3 served as loading control for cytoplasm and nucleus proteins respectively (B) UCHL1 induced the expression of cyclin D1 and uPA. $\beta$-actin served as loading control.

end of the experiment. In the remaining 4 mice, all of them developed lymph node metastases, accompanied with positive UCHL1 staining (Fig. 3A). However, no lymph node metastasis was found in HCT8/pcDNA3.1 group. The percentage of metastatic lymph nodes with respect to the total visible lymph nodes per mouse was significantly higher in the HCT8/ UCHL1 group (22\%) than in the control group $(0 \% ; \mathrm{P}<0.05)$ (Fig. 3B). Furthermore, UCHL1 re-expression dramatically promoted lung metastasis of HCT8 cells. The metastatic loci in the HCT8/UCHL1 group (58 \pm 30$)$ were significantly more than those in the control group $(7 \pm 6 ; \mathrm{P}<0.05)$ (Fig. 3C-D). These results indicated that UCHL1 promoted tumorigenesis and metastasis of HCT8 cells in vivo.

Deubiquitinase activity is crucial for UCHL1 to promote the malignant transformation of HCT8 cells. To investigate if the deubiquitinating capability is essential for UCHL1 function, we constructed a C90S mutant (with cysteine 90 converted to serine), with impaired deubiquitinase activity (17). Then the HCT8/UCHL1-C90S stable cell line was generated (Fig. 4A). The proliferation, migration, and invasion capabilities of HCT8/UCHL1-C90S cells were similar to those of HCT8/ pcDNA3.1 cells (Fig. 4B-D). These results indicated that the malignant transformation promoting function of UCHL1 was dependent on its deubiquitinating activity.

UCHL1 upregulates $\beta$-catenin by enhancing its stability. To see whether UCHL1 could affect $\beta$-catenin expression in HCT8 cells, we detected $\beta$-catenin in three stable cell lines. Our results confirmed that $\beta$-catenin expression was upregulated by UCHL1, but could not be upregulated by the UCHL1-C90S mutant (Fig. 5A).

To determine whether UCHL1 upregulates $\beta$-catenin expression by enhancing its stability, a half-life assay was performed. The data showed that UCHL1 re-expression significantly extended the half-life of $\beta$-catenin. In contrast, we failed to observe this function in the UCHL1-C90S mutant (Fig. 5B). These results indicated that UCHL1 upregulated $\beta$-catenin by enhancing its stability, depending on its deubiquitinating activity.

UCHL1 induces modification of proliferation, migration and invasion-related genes. To further confirm the effect of UCHL1 on $\beta$-catenin/TCF signaling, the immunoblotting analyses were carried out using the protein isolated from the cell cytoplasm and nucleus in three stable cell lines. Our 
results demonstrated that UCHL1 re-expression increased the accumulation of $\beta$-catenin in cytoplasm, which resulted in increased $\beta$-catenin translocation into cell nucleus (Fig. 6A). However, $\beta$-catenin remained unchanged in HCT8/UCHL1C90S cells compared with HCT8/pcDNA 3.1 cells.

Cyclin D1 and serine protease urokinase plasminogen activator (uPA) are transcriptionally induced by $\beta$-catenin in the nucleus $(18,19)$. As shown in Fig. $6 \mathrm{~B}$, both molecules were upregulated in HCT8/UCHL1 cells, but not in HCT8/pcDNA 3.1 and HCT8/UCHL1-C90S cells. These data suggested that UCHL1 activated the $\beta$-catenin/TCF signaling, depending on its deubiquitinating activity.

\section{Discussion}

Although much evidence indicates that UCHL1 is related to $\mathrm{CRC}$, the mechanism is still ambiguous. Some investigations have shown that UCHL1 methylation was more frequent in CRC tissues than in normal colorectal tissues $(9,10,20)$, whereas others have indicated that high UCHL1 expression was related to colorectal tumor progression, invasion, lymph node metastasis, and poor clinical outcome $(15,21-23)$. In this study, we confirmed our previous results by establishing that UCHL1 played an oncogenic role by positively regulating the proliferation, invasion, and metastasis of HCT8 in vitro and in vivo. Nail pad injection of stable HCT8/UCHL1 cells promoted tumor formation in nude mice. These data are consistent with that UCHL1 expression is related to tumor size and tumor stage in CRC $(15,16)$. In addition, tail vein injection of stable HCT8/UCHL1 cells promoted both lymph node and lung metastases in nude mice. These data are consistent with that UCHL1 expression is related to lymph node metastases in clinical colorectal patients $(16,23)$.

The Wnt $/ \beta$-catenin pathway is implicated in over $90 \%$ of colon cancers. The activation of $\beta$-catenin results in constitutive activity of $\beta$-catenin TCF transcriptional complex, which drives tumor formation (24). While exploring the mechanism by which UCHL1 promoted the malignant transformation of HCT8, we found that UCHL1 upregulated the expression of $\beta$-catenin by decelerating its degradation depending on its deubiquitinating activity. In agreement with our data, a recent study has also demonstrated that UCHL1 upregulated $\beta$-catenin signaling in 293 and NIH 3T3 cells (25). It is well known that the $\beta$-catenin subcellular distribution is greatly related to its oncogenic transcriptional regulation. The stabilized form of $\beta$-catenin accumulates in cytoplasm and is then translocated into the cell nucleus, where it carries out a pivotal role in tumorigenesis by activating the Wnt signaling target genes. In our investigation, UCHL1 induction increased the accumulation of $\beta$-catenin in the cytoplasm, which resulted in increased $\beta$-catenin translocation into the cell nucleus. Then upregulation of cyclin D1 and uPA were detected. Both cyclin D1 and uPA are target genes of $\beta$-catenin $(18,19)$. Cyclin D1 is a major regulator of the progression of cells into the proliferative stage of the cell cycle (26). The increased expression of cyclin D1 can stimulate CDK activity and promote the cell cycle progression. uPA is a protease involved in degradation of the extracellular matrix. Upregulation of uPA promotes tumor progression, particularly tumor invasion and metastasis $(27,28)$. These results agree with the cell behaviors that we have demonstrated in this study. Thus, we believe that UCHL1 promotes the malignant transformation of HCT8 by activating $\beta$-catenin signaling.

Several studies have demonstrated the importance of the deubiquitinating activity of UCHL1 in cancer progression and metastasis $(5,13,25)$. In our study, C90S, a mutant of UCHL1 with impaired deubiquitinating activity, did not induce the activation of $\beta$-catenin signaling and it did not promote the proliferation, migration, and invasion capabilities of HCT8 cells. However, identification of other target genes of UCHL1 deubiquitinating activity will provide more insight into the mechanisms of UCHL1 in oncogenesis in CRC.

The conflicting study results of UCHL1 function in CRC may be because of the inadequate sample and the exact subtype of CRC. Besides, UCHL1, which contains two opposing enzymatic activities, deubiquitinating enzyme activity and ubiquitin ligase enzyme activity, may function differently under certain circumstances. Thus, further study is needed to elaborate the exact role of UCHL1, to clarify the function of UCHL1 in the tumorigenic pathway and to assist in the development of novel biomarkers for early detection and accurate diagnosis.

In summary, we provide both in vitro and in vivo evidence that UCHL1 functions as an oncogene by increasing cell proliferation, migration, and invasion capabilities in HCT8 cells through the $\beta$-catenin/TCF pathway depending on its deubiquitinating activity.

\section{Acknowledgements}

This research was supported by the National Key Basic Research Program of China (2007CB914700). We are grateful to Dr Julia Shackelford (Lineberger Comprehensive Cancer Center, University of North Carolina at Chapel Hill, Chapel Hill, North Carolina, USA) for providing us with wild-type pcDNA3.1/UCHL1 plasmid.

\section{References}

1. Parkin DM, Bray F, Ferlay J and Pisani P: Global cancer statistics, 2002. CA Cancer J Clin 55: 74-108, 2005.

2. Levin B and Boyle P(eds): World cancer report 2008. International Agency for Research on Cancer, Lyon, pp374-379, 2008.

3. Osaka H, Wang YL, Takada K, Takizawa S, Setsuie R, Li H, Sato Y, Nishikawa K, Sun YJ, Sakurai M, et al: Ubiquitin carboxy-terminal hydrolase L1 binds to and stabilizes monoubiquitin in neuron. Hum Mol Genet 12: 1945-1958, 2003.

4. Setsuie R and Wada K: The functions of UCH-L1 and its relation to neurodegenerative diseases. Neurochem Int 51: 105-111, 2007.

5. Kim HJ, Kim YM, Lim S, Nam YK, Jeong J, Kim HJ and Lee KJ: Ubiquitin C-terminal hydrolase-L1 is a key regulator of tumor cell invasion and metastasis. Oncogene 28: 117-127, 2009.

6. Leiblich A, Cross SS, Catto JW, Pesce G, Hamdy FC and Rehman I: Human prostate cancer cells express neuroendocrine cell markers PGP 9.5 and chromogranin A. Prostate 67: 1761-1769, 2007.

7. Tezel E, Hibi K, Nagasaka T and Nakao A: PGP9.5 as a prognostic factor in pancreatic cancer. Clin Cancer Res 6: 4764-4767, 2000.

8. Li L, Tao Q, Jin H, van Hasselt A, Poon FF, Wang X, Zeng MS, Jia WH, Zeng YX, Chan AT and Cao Y: The tumor suppressor UCHL1 forms a complex with p53/MDM2/ARF to promote p53 signaling and is frequently silenced in nasopharyngeal carcinoma. Clin Cancer Res 16: 2949-2958, 2010.

9. Okochi-Takada E, Nakazawa K, Wakabayashi M, Mori A Ichimura S, Yasugi T and Ushijima T: Silencing of the UCHL1 gene in human colorectal and ovarian cancers. Int J Cancer 119: 1338-1344, 2006. 
10. Yu J, Tao Q, Cheung KF, Jin H,Poon FF, Wang X, Li H, Cheng YY, Röcken C, Ebert MP, et al: Epigenetic identification of ubiquitin carboxyl-terminal hydrolase L1 as a functional tumor suppressor and biomarker for hepatocellular carcinoma and other digestive tumors. Hepatology 48: 508-518, 2008.

11. Mandelker DL, Yamashita K, Tokumaru Y, Mimori K, Howard DL, Tanaka Y, Carvalho AL, Jiang WW, Park HL, Kim MS, et al: PGP9.5 promoter methylation is an independent prognostic factor for esophageal squamous cell carcinoma. Cancer Res 65: 4963-4968, 2005.

12. Miyoshi Y, Nakayama S, Torikoshi Y, Tanaka S, Ishihara H, Taguchi T, Tamaki Y and Noguchi S: High expression of ubiquitin carboxy-terminal hydrolase-L1 and -L3 mRNA predicts early recurrence in patients with invasive breast cancer. Cancer Sci 97: 523-529, 2006

13. Takase T, Hibi K, Yamazaki T, Nakayama H, Taguchi $M$, Kasai Y, Ito K, Akiyama S, Nagasaka T and Nakao A: PGP9.5 overexpression in esophageal squamous cell carcinoma. Hepatogastroenterology 50: 1278-1280, 2003.

14. Wang WJ, Li QQ, Xu JD, Cao XX, Li HX, Tang F, Chen Q, Yang JM, Xu ZD and Liu XP: Overexpression of ubiquitin carboxy terminal hydrolase-L1 induces apoptosis in breast cancercells. Int J Oncol 33: 1037-1045, 2008.

15. Yamazaki T, Hibi K, Takase T, Tezel E, Nakayama H, Kasai Y, Ito K, Akiyama S, Nagasaka T and Nakao A: PGP9.5 as a marker for invasive colorectal cancer. Clin Cancer Res 8: 192-195, 2002.

16. Ma Y, Zhao M, Zhong J, Shi L, Luo Q, Liu J, Wang J, Yuan X and Huang C: Proteomic profiling of proteins associated with lymph node metastasis in colorectal cancer. J Cell Biochem 110: $1512-1519,2010$.

17. Larsen CN, Price JS and Wilkinson KD: Substrate binding and catalysis by ubiquitin C-terminal hydrolases: identification of two active siteresidues. Biochemistry 35: 6735-6744, 1996.

18. Hiendlmeyer E, Regus S, Wassermann S, Hlubek F, Haynl A, Dimmler A, Koch C, Knoll C, van Beest M, Reuning U, et al: Beta-catenin upregulates the expression of the urokinase plasminogen activator in human colorectal tumors. Cancer Res 64: 1209-1214, 2004.
19. Shtutman M, Zhurinsky J, Simcha I, Albanese C, D'Amico M, Pestell R and Ben-Ze'ev A: The cyclin D1 gene is a target of the $\beta$-catenin/LEF-1 pathway. Proc Natl Acad Sci USA 96: 5522-5527, 1999.

20. Fukutomi S, Seki N, Koda K and Miyazaki M: Identification of methylation-silenced genes in colorectal cancer cell lines genomic screening using oligonucleotide arrays. Scand J Gastroenterol 42: 1486-1494, 2007

21. Akishima-Fukasawa Y, Ino Y, Nakanishi Y, Miura A, Moriya Y, Kondo T, Kanai Y and Hirohashi S: Significance of PGP9.5 expression in cancer-associated fibroblasts for prognosis of colorectal carcinoma. Am J Clin Pathol 134: 71-79, 2010.

22. Hong Y, Ho KS, Eu KW and Cheah PY: A susceptibility gene set for early onset colorectal cancer that integrates diverse signaling pathway: implication for tumorigenesis. Clin Cancer Res 13: 1107-1114, 2007.

23. Mizukami H, Shirahata A, Goto T, Sakata M, Saito M, Ishibashi K, Kigawa G, Nemoto H, Sanada Y and Hibi K: PGP9.5 methylation as a marker for metastatic colorectal cancer. Anticancer Res 28: 2697-2700, 2008.

24. Bienz $M$ and Clevers H: Linking colorectal cancer to Wnt signaling. Cell 103: 311-320, 2000.

25. Bheda A, Yue W, Gullapalli A, Whitehurst C, Liu R, Pagano JS and Shackelford J: Positive reciprocal regulation of ubiquitin C-terminal hydrolase L1 and beta-catenin/TCF signaling. PLoS One 4: e5955, 2009.

26. Sherr CJ: Cancer cell cycles. Science 274: 1672-1677, 1996.

27. Mazaud S, Guyot R, Guigon CJ, Coudouel N, Le MagueresseBattistoni B and Magre S: Basal membrane remodeling during follicle histogenesis in the rat ovary: contribution of proteinases of the MMP and PA families. Dev Biol 277: 403-416, 2005.

28. Morgan H and Hill PA: Human breast cancer cell-mediated bone collagen degradation requires plasminogen activation and matrix metalloproteinase activity. Cancer Cell Int 5: 1, 2005. 